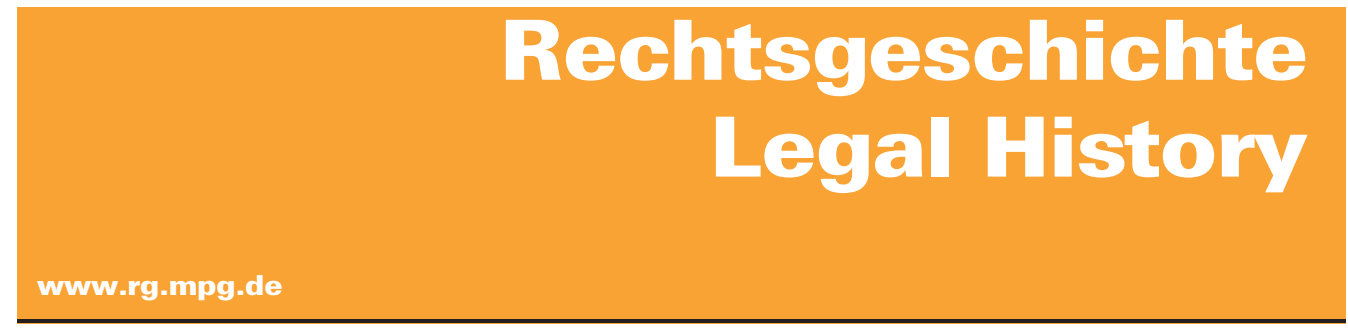

http://www.rg-rechtsgeschichte.de/rg24

$\operatorname{Rg} 242016$

$361-363$

Zitiervorschlag: Rechtsgeschichte - Legal History Rg 24 (2016)

http://dx.doi.org/10.12946/rg24/361-363

\title{
Ute Dercks
}

\section{Kapitellskulptur des 12./13. Jahrhunderts in multimedialer Vision}




\section{Ute Dercks \\ Kapitellskulptur des 12./13. Jahrhunderts in multimedialer Vision}

Die Kunstgeschichte ist mehr als jede andere geisteswissenschaftliche Disziplin eine an das Abbild gebundene Wissenschaft. Standen vor 1839 dem Jahr der Veröffentlichung verschiedener Experimente $\mathrm{zu}$ fotografischen Verfahren - den Kunstinteressierten lediglich das eigene Gedächtnis, Skizzen oder Reproduktionsgrafiken zur Verfügung, gestattete erst die Fotografie mit ihrem visuellen Gedächtnis die für die Kunstgeschichte grundlegende Methode des vergleichenden Sehens, d. h. vergleichende Studien anzustellen zu nicht am selben Ort befindlichen Kunst- oder Bauwerken. Wenn auch die Diskussion um die Objektivität von Fotografien einen eigenen Diskurs verlangt, kann ohne weiteres behauptet werden, dass sich die Kunstgeschichte erst durch die Fotografie und die entsprechenden Projektionsverfahren als eigenes Fach etablierte - gleichermaßen kann aber auch von einem durch die Fotografie geprägten kunsthistorischen Blick gesprochen werden, der an die Abbildungs- und Wiedergabetechniken gebunden ist.

Den technischen Fortschritt nutzend machte sich die Kunstgeschichte neben der Fotografie auch die Anwendung physikalisch-technischer Instrumente wie Röntgengeräte oder Computertomographen zu eigen, die ohne das Objekt zu beschädigen unter der Oberfläche Liegendes wie etwa Untermalungen eines Bildes oder die Armierung einer Gipsfigur zum Vorschein bringen und so einen Werkprozess wie Pentimenti, Entwurfsskizzen oder unterschiedliche Versionen und Zustände eines Kunstwerks vor Augen führen. Zu den traditionellen analogen und elektronischen bildgebenden Verfahren sowie deren Katalogisierung - nicht nur darin ist die bildbasierte Wissenschaft untrennbar mit der textbasierten verbunden - traten digitale Arbeitsinstrumente, die eine Visualisierung unabhängig von Zeit und Raum des Originalstandorts eines Kunst- oder Bauwerks in Größe und Farbe variabel gleichzeitig an mehreren Orten verfügbar machen. Es entstehen Konstruktionsmodelle nach Plänen von ungebauter oder hypothetischer Architektur, von nicht realisierten Denkmälern und Kunstgegenständen, Zusammenstellungen von historischen, nicht mehr existierenden
Komplexen, temporäre Installationen wie etwa Ausstellungen - das Spektrum der Anwendungen reicht von der Sichtbarmachung von Verborgenem bis hin zur Erschaffung von Scheinobjekten, die jenseits der Kohlenstoff-Welt erzeugt werden.

Eine solche Visualisierung ist Ausgangspunkt des webbasierten, frei zugänglichen Projektes CENOBIUM, das ein vom Kunsthistorischen Institut in Florenz (Max-Planck-Institut) und dem Istituto di Scienza e Tecnologie dell'Informazione, Consiglio Nazionale delle Ricerche in Pisa (ISTI/ CNR) mit verschiedenen internationalen Kooperationspartnern entwickeltes Projekt zur Erforschung mittelalterlicher Bauskulptur ist (http:// cenobium.isti.cnr.it). Im Fokus stehen Kreuzgänge mit Zyklen vor allem historisierter Kapitelle, d. h. von reliefierten Säulenkapitellen mit narrativen Darstellungen. Diese meist biblischen, hagiographischen oder allegorischen Erzählungen legen sich dabei in mehreren Szenen um den Kapitellkern herum, so dass sich die Geschichten nur in der Abfolge, also beim physischen Umrunden der Säule, nachvollziehen lassen. Mittels moderner Technologien wie hochauflösender Digitalfotografie, 3D-Scanning sowie einer interaktiven Webanwendung werden Barrieren wie die Entfernung zum Objekt, ungünstige Lichtverhältnisse, architektonisch bedingte Hindernisse oder auch konservatorische Schutzmaßnahmen überwunden, die das Studium der Kapitelle vor Ort behindern. Diese Technologien ermöglichen es, die Bauskulptur in ihrer Oberflächenstruktur, Farbe, Materialtextur und Reliefbearbeitung so zu studieren, wie sie die Bildhauer selbst vor Augen hatten und wie sie gleichzeitig im architektonischen Zusammenhang stehen. Nicht nur für kunsthistorische Fragestellungen, sondern auch für die Konservierung und Restaurierung der Kapitelle, die im offenen Kreuzgang allen Umwelteinflüssen ausgesetzt sind, ist das Projekt ein nützliches Hilfsmittel, da durch die Fotografien nicht nur Materialdetails sichtbar gemacht werden, sondern die 3D-Modelle mittels eines die Oberfäche abtastenden Laserscanners eine virtuelle Form erschaffen, die sich mit zukünftig gemachten 3D-Konstruktionen vergleichend messen lassen. 


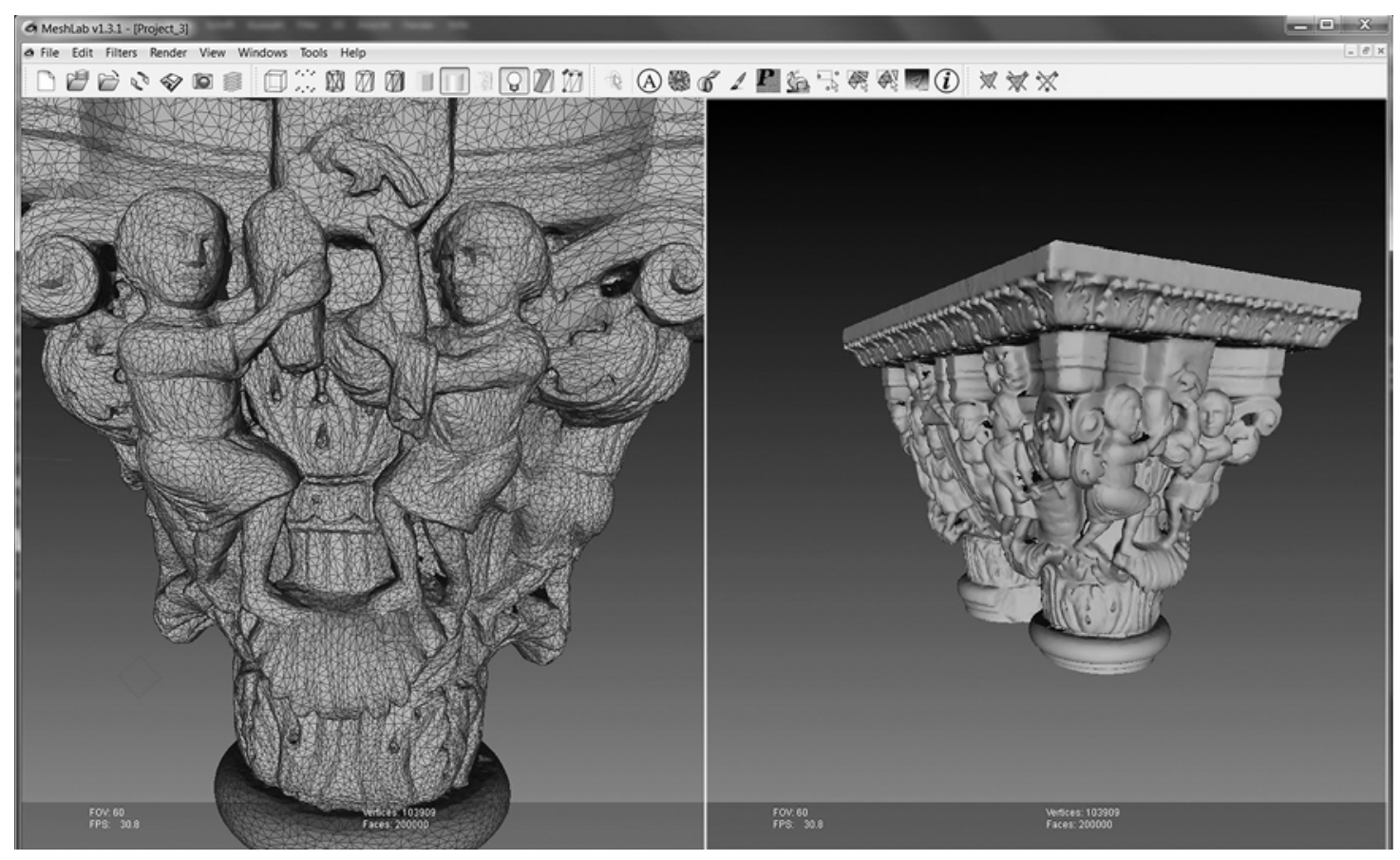

3D-Modell des Genesiskapitells aus dem Kreuzgang von Monreale, visualisiert mit der Software Meshlab (@ KHI-MPI / ISTI-CNR)

Die Anordnung der Kapitelle in den jeweiligen Kreuzgängen ist auf der Basis des Grundrisses in eine schematische Grafik umgesetzt, so dass die Erkundung und Besichtigung intuitiv erfolgen kann oder über den Begleittext, der das Dargestellte erklärt und zusammen mit den transkribierten Inschriften durch Keywords verschlagwortet und auffindbar macht.

Durch die Kombinationen der verwendeten Technologien, die nicht nur eine Nahsichtigkeit, sondern auch die Allansichtigkeit mehrerer Kapitelle erlauben, wird eine Art der Analyse entfaltet, die vor Ort im Kreuzgang nicht möglich ist, und woraus sich ein breites Anwendungsprofil des Projektes ergibt für die Forschung, Lehre und Denkmalpflege, ebenso wie in musealen oder didaktischen Zusammenhängen.

Aber so wie die Fotografie kein Ersatz für das Studium des Kunstwerks vor Ort ist, so ersetzt auch das 3D-Modell nicht das reale Objekt; stattdessen geht es vielmehr darum, der Forschung ein zusätzliches, wenn nicht sogar ein anderes Instrument zu Verfügung zu stellen.

CENOBIUM ist als work-in-progress konzipiert, dessen Integration neuer Technologien und Inhalte Teil des Programms sind und auf der Zusammenarbeit zweier unterschiedlicher Disziplinen, der Kunstgeschichte und der angewandten Informatik, fußt. Impulse aus der Forschung beider Fachrichtungen werden gegenseitig aufgenommen. Das Innovationspotenzial liegt daher im beiderseitigen Interesse der Partner, um aus der Inspiration und den Möglichkeiten des anderen neue Ideen zu schöpfen. Die Ausbauschritte und Verbesserungen laufen schubweise, der Kreis der Kreuzgänge sowie die Art der Anwendungen und Tools werden kontinuierlich erweitert, um eine funktionierende virtuelle Forschungsumgebung weiterzuentwickeln. 
Forum forum

\section{Bibliographie}

- Literatur zum Projekt in Auswahl (alle als pdf verfügbar auf http://cenobium.isti.cnr.it/monreale/links):

- Corsini, M., M. Dellepiane, U. Dercks, F. Ponchio, M. Callieri, D. Keultjes, A. Marinello, R. Sigismondi, R. Scopigno, G. Wolf (2010), CENOBIUM - Putting together the Romanesque Cloister Capitals of the Mediterranean Region; in: Space, Time, Place. Third International Conference on Remote Sensing in Archaeology, Tiruchirappalli, Tamil Nadu, India 2009, ed. by S. Campana, M. Forte, C. Liuzzain, Oxford, 189-194

- Dercks, U., F. Ponchio, R. Scopigno (2012), CENOBIUM - A project for the Multimedia Representation of Romanesque Cloister Capitals in the Mediterranean Region; in: EVA 2012 Berlin (Elektronische Medien \& Kunst, Kultur, Historie), ed. by A. Bienert, F. Weckend, J. Hemsley, Berlin, 78-83

- Salonia, P., S. Scolastico. A. Marcolongo, T. Leti Messina (2009), Survey and 3D reconstruction of the St. Orso capitals in Aosta, through three-focal photogrammetry; in: Virtual Systems and Multimedia, (VSMM 2009). 15th International Conference, Prague, $35-40$

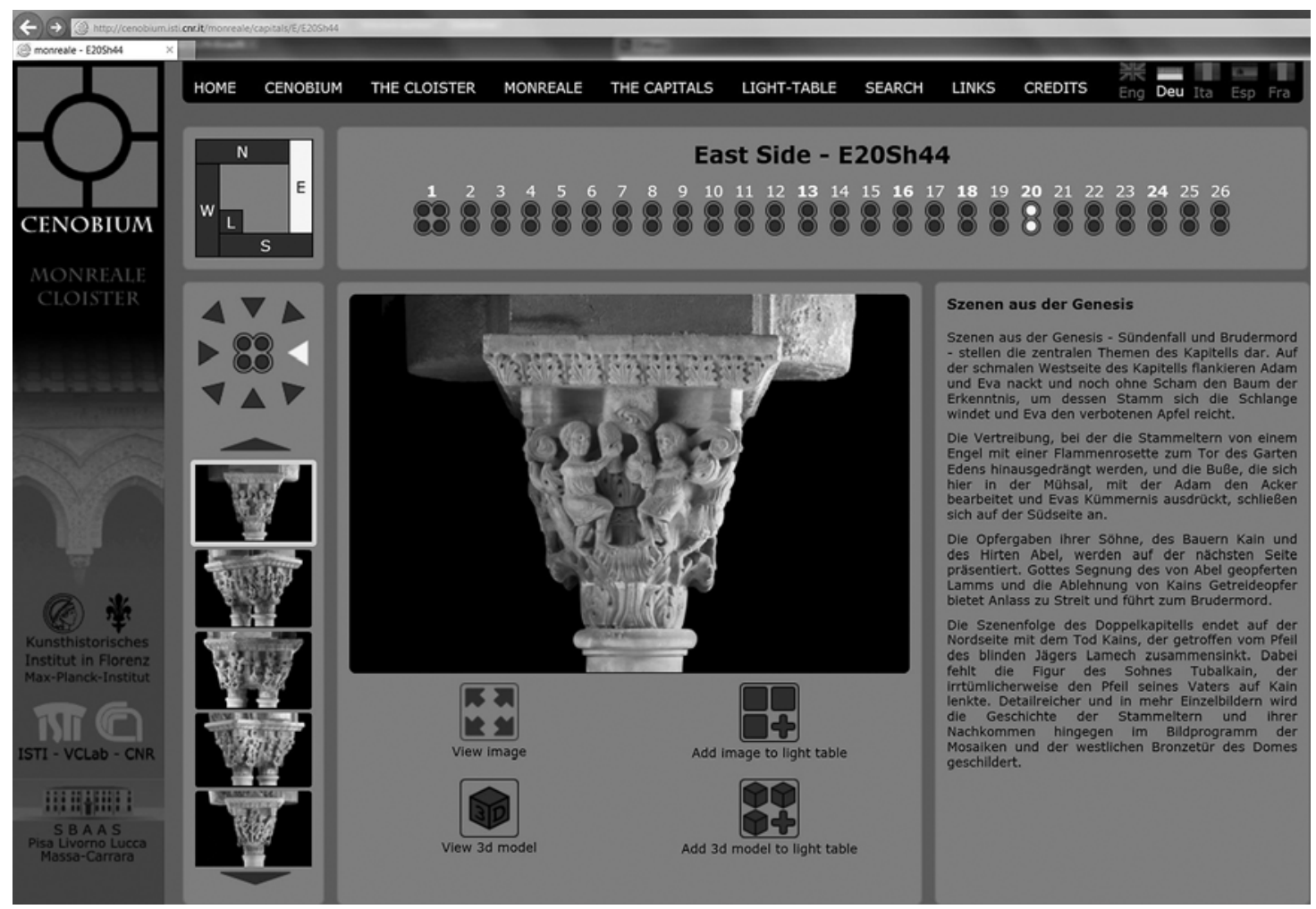

Genesiskapitell aus dem Kreuzgang von Monreale, auf der Webseite CENOBIUM (@ KHI-MPI / ISTI-CNR) 